\title{
Circuit
}

Musiques contemporaines

\section{La création électroacoustique et les jeunes... Une démarche sur mesure}

\section{Roxanne Turcotte}

Volume 16, numéro 2, 2006

Musique de création et jeunes publics

URI : https://id.erudit.org/iderudit/902399ar

DOI : https://doi.org/10.7202/902399ar

Aller au sommaire du numéro

Éditeur(s)

Les Presses de l'Université de Montréal

ISSN

1183-1693 (imprimé)

1488-9692 (numérique)

Découvrir la revue

Citer cet article

Turcotte, R. (2006). La création électroacoustique et les jeunes... Une démarche sur mesure. Circuit, 16(2), 73-82. https://doi.org/10.7202/902399ar d'utilisation que vous pouvez consulter en ligne.

https://apropos.erudit.org/fr/usagers/politique-dutilisation/ 


\title{
La création électroacoustique et les jeunes... Une démarche
}

\section{sur mesure}

\author{
Roxanne Turcotte
}

La création musicale en studio est un jeu d'enfant. La musique électroacoustique est-elle inaccessible? Intouchable? Il s'agit plutôt d'un incontournable. Selon l'approche compositionnelle choisie, il est possible de rejoindre les enfants par les sensations magiques qu'elle procure. L'appréciation de cette musique par les jeunes est facilitée par l'impression étrange produite par des sonorités et un style encore mal connus et sous-utilisés dans les institutions. De toute évidence, un parallèle existe entre une piste d'effets sonores cinématographiques et l'approche musicale électroacoustique. Si on compare la structure et les sonorités d'abord descriptives, puis imaginaires, on remarque des similitudes au niveau des dynamiques et des timbres diffusés dans un système en multicanaux où règne une ambiance accrocheuse. Sans l'image, le mixage devient une œuvre musicale. La musique peut nous surprendre et les enfants connaissent bien l'effet du son issu du cinéma, de la télévision, d'Internet et de leur console de jeux électronique. Peu de spécialistes en enseignement de la musique ont les outils nécessaires pour intégrer adéquatement la création sonore électroacoustique dans leur programme. Faute de matériel didactique et technologique, il leur faut puiser dans les ressources à l'état brut : les œuvres.

Le développement de l'imaginaire chez le jeune lui permet de s'épanouir tout en s'amusant. La musique doit décrire un univers, un paysage ou un récit réel ou irréel qu'il peut capter et décoder aisément. Une allure musicale s'adapte au scénario qu'il organise dans ses pensées. Des éléments référentiels 
forment le langage intelligible des ambiances : inversion des rôles du son et de l'image au cinéma, impressionnisme, littérature fantastique et étrange, monde de rêve et d'illusion parfaite d'un Walt Disney, contes et légendes du monde entier décrivent bien un univers sonore aux mille reflets.

La participation créative des enfants lors de la composition d'une œuvre apporte une touche particulière à un projet. Ils ont la surprise de s'entendre et se sentent impliqués, donc plus réceptifs aux sons qui parlent par eux-mêmes.

La création électroacoustique est l'effet poétique d'une émotion puisée dans l'organisation des sons, la spontanéité de l'exécution et les rythmes viscéraux. Tout devient un matériau sonore palpable. L'improvisation et l'interactivité permettent des choix et lèguent des impressions. L'humour et le rêve restent toujours la base de l'accessibilité à ce qui semblait inapprochable. Une musique sans frontières implique la combinaison de différentes expressions musicales et de sources d'inspiration nombreuses où tout s'imbrique avec subtilité et nuances. Des éléments enfantins donnent un souffle de vie, une jeunesse constante, une immortalité et une ouverture d'esprit; en un mot, un vent de fraîcheur!

\section{Démarche électroacoustique et apprentissage musical}

Message véhiculé pour les adultes, apprentissage pour les enfants, il semble que l'expression artistique prenne souvent une forme éducative. Toutefois, les œuvres nous atteignent sans nécessairement laisser des traces. Des notions sont apprises à notre insu lors d'une production.

Les phénomènes sonores croisent l'univers des sciences, ce qui permet de concrétiser la musique. Le passage vers l'abstraction s'effectue spontanément et en douceur. L'objet à l'origine du son est une explication plausible de sa provenance et une porte d'entrée pour les découvertes auditives et l'initiation musicale. Des cordes se mettent en vibration, une peau résonne sous la main d'un interprète, un bidule sonore inventé, imaginé et recherché fait son apparition. Dans le cadre d'une initiation musicale, l'assimilation des modes de production du son est primordiale, ainsi que celle du rôle de l'air et de l'onde produite, de la spatialisation et des effets naturels ou synthétiques. Ces éléments définissent le langage musical dans son ensemble tout en servant la création.

\section{Création d'une œuvre et des matériaux qui la composent... L'art de s'amuser !}

L'électroacoustique est un jeu, un bricolage, un casse-tête ou une jonglerie de bruits : telle est la perception des enfants lorsqu'on leur met un objet sonore entre les mains. Ils utilisent leur intuition pour générer des ambiances et ainsi, sortir de l'ordinaire! 
Un nombre considérable d'analogies peuvent être employées pour alléger des notions qui relèvent de la physique : l'écho des montagnes et le fonctionnement d'un radar, la réverbération d'un gymnase, les timbres naturels... Différents paramètres acoustiques prennent place dans la structure d'une composition. Des cordes vibrantes de tensions variées; des bidules à vent fabriqués avec des bouteilles et des ballons que l'on dégonfle doucement, laissant s'échapper un son très aigu; différents matériaux frappés en guise de percussion. Une boîte musicale ou un piano mécanique évoquent la technologie du séquenceur. L'effet Doppler ${ }^{1}$ est illustré par un tuyau agité très rapidement ou une vocalise descendante.

L'amplification est représentée par des bidules à vent de forme conique, fabriqués par les enfants. Ils font l'expérience d'émettre des sons dans une boîte ou dans la caisse de résonance d'un instrument à lames. Un long tuyau de PVC peut servir de didjéridou pour le chant des harmoniques. Une plaque de métal au fond d'un contenant peut servir de chambre réverbérante. Des effets sonores surprenants peuvent être tirés d'une casserole remplie d'eau qu'on agite et qu'on frappe avec un maillet. Les timbres ont des textures lisses, granuleuses, sèches, liquides, résonantes, qui déboulent par grappes, et ils peuvent être obtenus à l'aide d'objets divers. Ils se propagent en voyageant dans une petite bulle d'air qui se déplace à la vitesse du vent et peuvent parfois rencontrer des obstacles, les effleurer ou rebondir comme un ballon ainsi qu'un retour d'écho à l'effet «boomerang». Le jeu du téléphone est une façon simple de comprendre le phénomène de la propagation des sons.

L'alimentation électrique de certains instruments fait passer un signal à travers un réseau. C'est l'image d'un tunnel, d'un passage souterrain ou d'un insecte bruyant qui s'y promène. On peut fabriquer un télégraphe à l'aide d'une pile sèche de type $\mathrm{D}$, d'un bloc de bois, de clous, de couvercles de boîte de conserve et de filage électrique. Le silence en musique est un effet de «vide » réalisable. Faire tinter une cloche ou tout autre objet dans un contenant vidé de son air : l'objectif est de faire prendre conscience aux enfants que sans air, il n'y a pas de son. Le vide est un moment de répit, c'est la respiration en musique. Une fois ces éléments rassemblés, une création prend son envol.

\section{Projets de spectacles, ateliers pour jeune public et composition musicale}

Certaines de mes musiques ont subi l'influence des concepts d'ateliers donnés aux jeunes enfants, aux adolescents ou même parfois aux adultes qui avaient simplement besoin d'une initiation aux technologies musicales à réinvestir dans une approche personnelle ou pour une utilisation en classe avec des élèves.
1. L'effet Doppler est le phénomène physique (acoustique et optique) à l'origine du décalage entre la longueur d'onde à l'émission et la longueur d'onde à la réception lorsque l'émetteur et le récepteur sont en mouvement l'un par rapport à l'autre. Dans le cas de l'onde sonore, cela signifie que la hauteur du son émis par une source en mouvement est perçue différemment par l'auditeur selon que la source s'en rapproche (le son perçu sera alors plus aigu que le son émis, et deviendra de plus en plus aigu jusqu'à ce que la source arrive au niveau de l'auditeur) ou s'en éloigne (auquel cas le son semblera devenir plus grave qu'il ne l'est en réalitê). (Note de la rédaction) 
Après que j'eus observé la réceptivité des jeunes, mon travail de création a pris une tournure figurative et presque impressionniste. L'électroacoustique fournit des repères où la structure harmonique des timbres offre un grand nombre de possibilités inventives. Malgré les apparences trompeuses, la musique électroacoustique rejoint la spontanéité de la musique viscérale que l'on retrouve dans les musiques traditionnelles du monde et les musiques populaires en général. La musique électroacoustique n'a pas de règles préétablies ni de concepts sculptés et destinés à l'intellect sans émotions. Cette approche relève souvent de «l'étrange", d'un monde magique réalisé à partir de sons environnementaux intrigants. Les adultes s'y perdent parfois, dans ce monde où Alice dissipe les repères du pays des merveilles.

\section{Sons et tintamarre :}

miniatures enfantines pour jeune public en cinq mouvements

Le spectacle, présenté dans le cadre de la série «Concertino» des Jeunesses Musicales du Canada en 1994, contribue à l'initiation des jeunes à la musique contemporaine, essentiellement à l'électroacoustique. Une version de la pièce est adaptée aux différents groupes d'âges et convertie en atelier offert dans le cadre des «Artistes à l'école» (Culture et éducation) depuis une dizaine d'années. Les enfants explorent le domaine des technologies musicales par la voie de la création. Les plus vieux travaillent en laboratoire informatique.

Sons et tintamarre est un conte sonore destiné aux enfants, ainsi qu'aux plus grands désireux de caresser leur imagination d'antan. Une histoire sonore dépeint les étapes de fabrication d'une œuvre électroacoustique dans l'atelier du compositeur. Des pièces minuscules sont tirées d'un concept musical faisant appel à une série de sons indépendants, ainsi qu'à de petites cellules rythmiques interprétées en direct sur scène, avec la participation du jeune public. De petites musiques sont enfermées dans une boîte musicale magique. Complice avec les enfants, un personnage appelé Tarapatapom nous plonge dans un univers de sons captés, échantillonnés, synthétisés, transformés, rythmés, façonnés et organisés en mouvements dans le temps et l'espace. Des enfants prêtent leur voix aux jeux et aux mouvements de la pièce, ultérieurement modifiée. Lors de la diffusion, ils se reconnaissent et c'est très drôle! 


\begin{tabular}{|l|l|}
\hline Synopsis & Mes sons \\
Tarapatapom vit dans un pays où poussent des & Ballon, jouets, voix d'enfants \\
sons bizarres. Elle rencontre Tintamarre, une & et transformations... \\
boîte musicale parlante qui lui fait connaître & \\
Coquet-le-Perroquet-qui-mémorise-tout. & \\
Ensemble, ils partent à la découverte du monde \\
de la création électroacoustique. Tous les sons \\
découverts sont mémorisés, traités, et gardés \\
dans la boîte magique. Après une écoute \\
attentive, les sons s'envolent pour créer des \\
ambiances sonores surprenantes. \\
$\begin{array}{l}\text { Ouvrez grandes vos oreilles! Ne laissez pas } \\
\text { s'échapper les sons avant de les avoir écoutés. }\end{array}$ \\
\hline Mes moyens sonores = \\
\hline
\end{tabular}

Jeu de cache-cache et spatialisation, magie d'une illustration sonore représentée par un montage serré, musique d'animation sans l'image, voyage d'un ballon sauteur aux effets rythmiques et stéréophoniques, manipulation, égalisation, renversements, montage... Un objet devenu quelque chose d'autre $! 8,9,10 \ldots$ des sons à dormir debout, Histoire sans paroles, Jouets, Ballon rouge qui bouge... L'œuf magique...

\begin{tabular}{|lllllll|}
\hline 00 & 10 & 20 & 30 & 40 & 50 & $60 \ldots$ \\
\hline
\end{tabular}

Exemple d'un travail de création sonore chez les jeunes à partir du modèle de Sons et tintamarre : expérimenter pour apprécier!

L'atelier débute par la présentation de l'œuvre électroacoustique. Les participants sont divisés en équipes de quatre et circulent à travers différentes installations.

La première étape consiste à créer un ancrage par l'élaboration d'un scénario sur un thème connu de l'enfant : ses repères. Les plus jeunes dessinent les séquences de l'histoire. Les plus vieux fournissent une description écrite des sons choisis. Les sources d'inspiration peuvent prendre différentes formes : synopsis, bande dessinée, dessin animé, clip, image, thème... Dans un deuxième temps, ils définissent leurs moyens sonores : instruments acoustiques, ordinateur, synthétiseur, sons concrets, voix... L'approche finale consiste en l'exécution en direct sur les images d'une vidéo. On procède ensuite au mixage et au montage. Lorsqu'on écoute le son sans l'image, le résultat est surprenant! 
Planche de travail \#1 - Un animal dans la ville

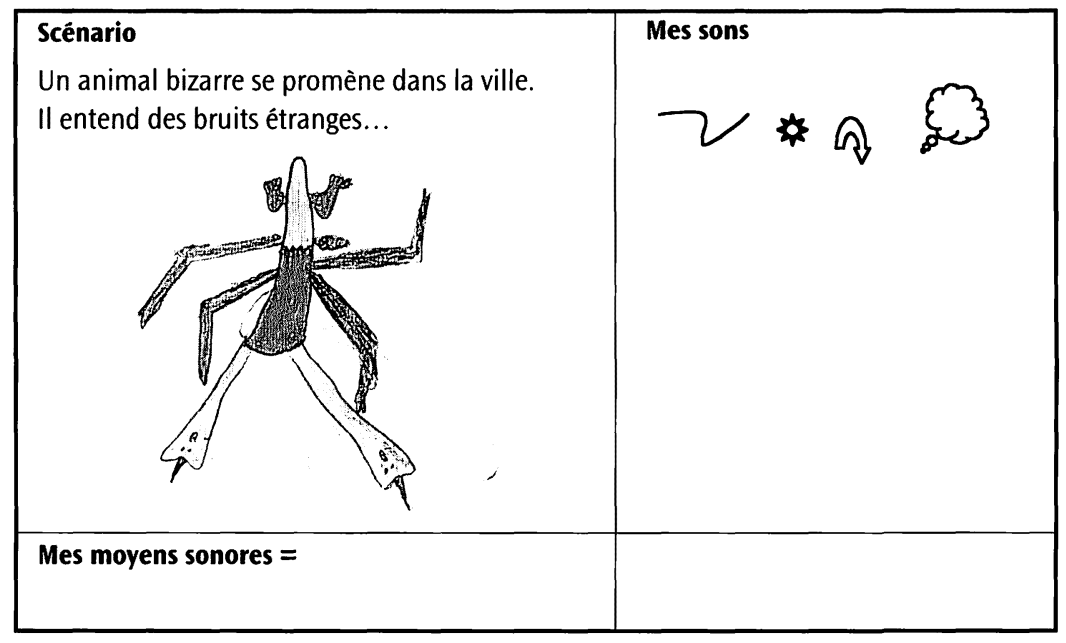

Partition d'écoute : la structure

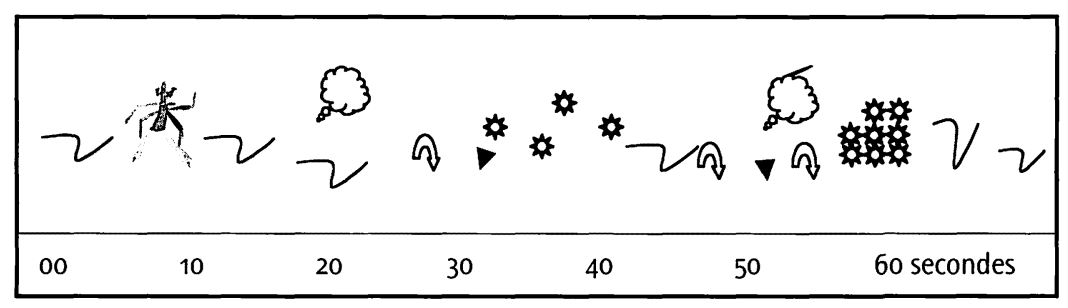

Partition d'écoute : structurer la pièce en disposant les sons dans un ordre précis sur la partition minutée.

\section{Coloriage sonore}

La sonorisation d'une histoire rejoint la pureté d'un dessin qui est un repère inné chez les enfants. On y ajoute des couleurs (timbres), une maquette (structure) et des plans détaillés (langage). 


\begin{tabular}{|l|l|l|}
\hline $\begin{array}{l}\text { Un voyage... } \\
\text { Décris ton thème }\end{array}$ & $\begin{array}{l}\text { Sons animés... } \\
\text { Décris ton thème }\end{array}$ & $\begin{array}{l}\text { Radio étudiante... } \\
\text { Décris ton thème }\end{array}$ \\
\hline & & \\
\hline Choix de sons & Choix de sons & Choix de sons \\
\hline $\begin{array}{l}\text { Odyssée de l'espace... } \\
\text { Décris ton thème }\end{array}$ & $\begin{array}{l}\text { Maison hantée... } \\
\text { Décris ton thème }\end{array}$ & $\begin{array}{l}\text { Une publicité... } \\
\text { Décris ton thème }\end{array}$ \\
\hline Choix de sons & Choix de sons & Choix de sons \\
\hline
\end{tabular}

Banque de sons de base et séquences à écouter et/ou utiliser : dresser une liste d'échantillons, de séquences et de sons synthétiques à écouter et sélectionner selon le thème choisi et la direction que prend l'œuvre.

Exemple :

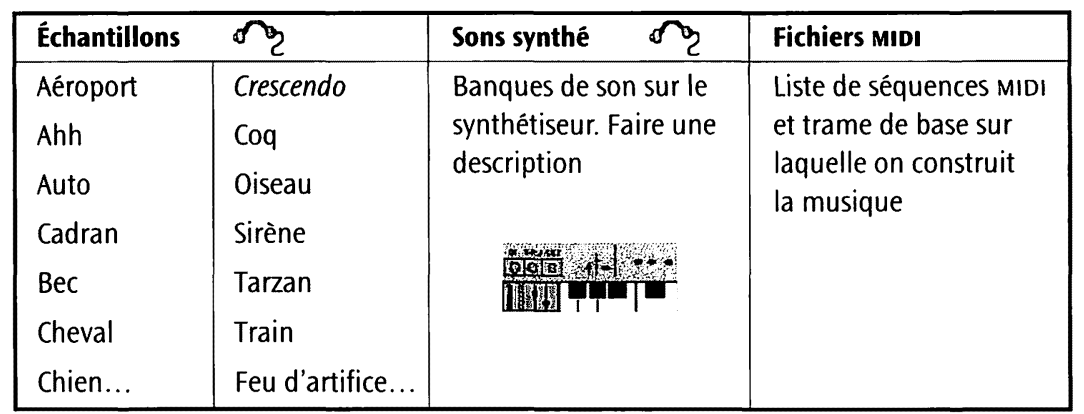

Minisérie (1990) : Trois petits scénarios sans mots et sans images

Cette œuvre a souvent fait l'objet d'essais chez des jeunes de niveau collégial ou secondaire, bien qu'elle ne leur soit pas spécifiquement destinée. Elle est très suggestive et évoque, pour certains enfants, une soirée d'Halloween, et pour d'autres, un monde d'extra-terrestres... Un environnement sonore autour d'une poursuite à travers différents contextes dans un univers à la Hitchcock; les saveurs colorées d'un Spielberg; une construction cinématographique pour les oreilles et l'imagination. Empreint de terreur, ce récit sonore raconte une 


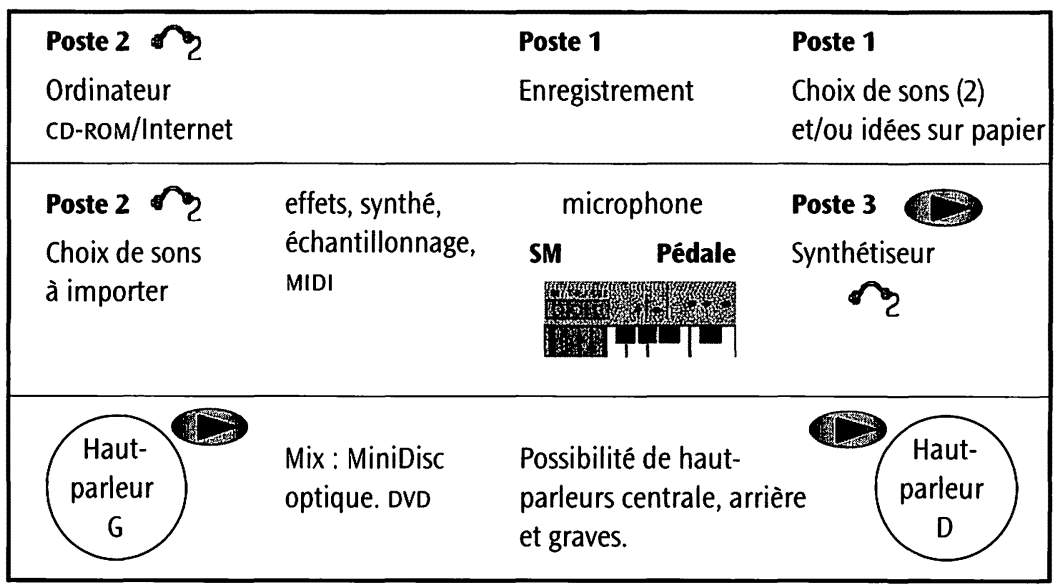

histoire macabre sur un ton plutôt humoristique, marqué par les changements rapides de plans d'une musique en trois dimensions. Les enfants écoutent, puis donnent leurs impressions sur ce qu'ils ont entendu. Chacun distingue les scénarios d'une façon particulière. Ils inventent leur propre histoire à partir de leur perception personnelle.

\section{Libellune (2003)}

Contraction de «libellule» et "lune», représentation du rêve et de l'évasion vers la lune, ce titre vient d'un mot inventé par ma fille Fannie. Les enfants en

\begin{tabular}{|l|l|}
\hline Synopsis & Mes sons \\
Parcours sonore d'une libellule (symbole de l'île & Instruments à vent modifiés \\
de Hondo au Japon) devenue nocturne un soir de & \\
pleine lune. Le bruissement de ses ailes, signe \\
de liberté et d'évasion, nous transporte dans \\
des univers fantastiques et vers différentes cultures \\
du monde en passant par des océans, des volcans \\
tranquilles et l'espace. Son vol est d'une telle \\
puissance que rien ne l'empêche de passer. Son \\
élégance est d'une légèreté et d'une pureté qui \\
se marient parfaitement avec le rayon de lune \\
légèrement doré. Le soir, tout est si tranquille! \\
Les sons deviennent parfaitement audibles. C'est \\
le meilleur moment pour la réflexion. \\
\hline Mes moyens sonores =
\end{tabular}


bas âge déforment souvent les mots, qui deviennent ainsi une création en soi et une grande source d'inspiration.

Libellune est un survol sonore dans les domaines du rêve et de l'imaginaire, aux sonorités empreintes de poésie, d'espace, de lieux, de personnages fantastiques et d'impressions aux couleurs intimistes.

Le canevas de structuration de l'œuvre demeure le même².

2. Voir le tableau de la page précédente

(p. 80). (Note de la rédaction)

Extrait de Fantaisie urbaine, 2005

Fantaisie urbaine - Partition d'écoute

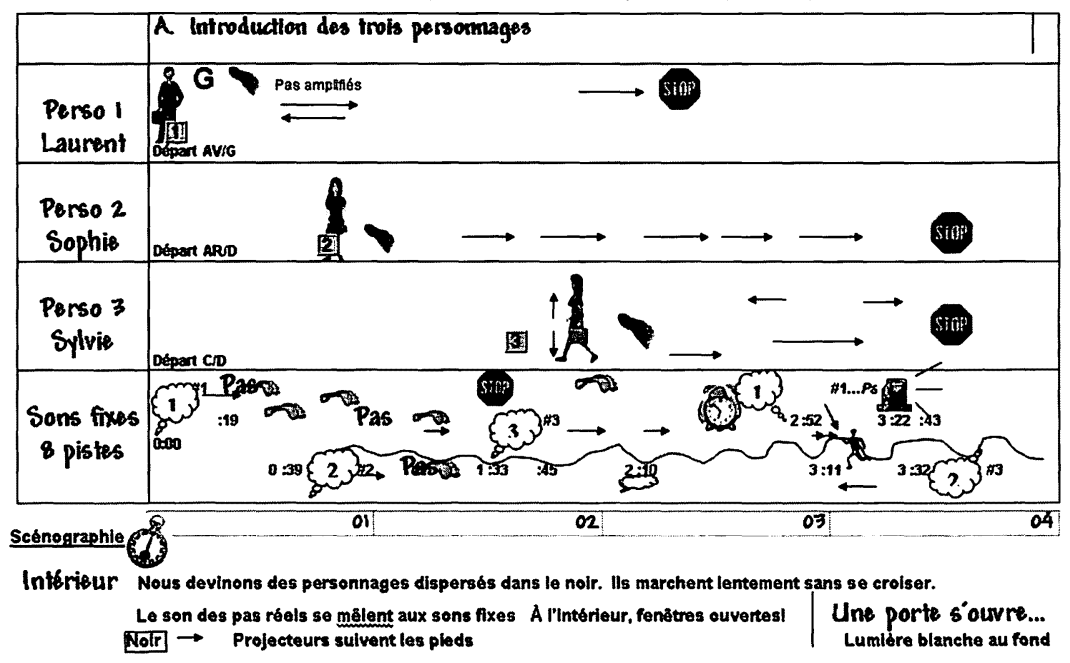

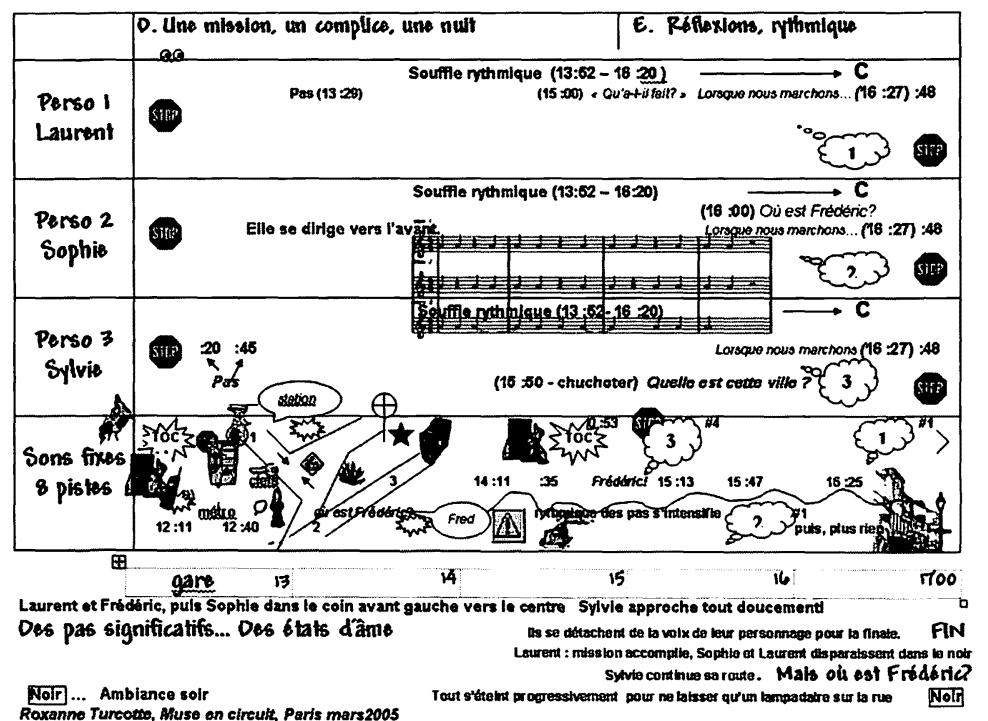




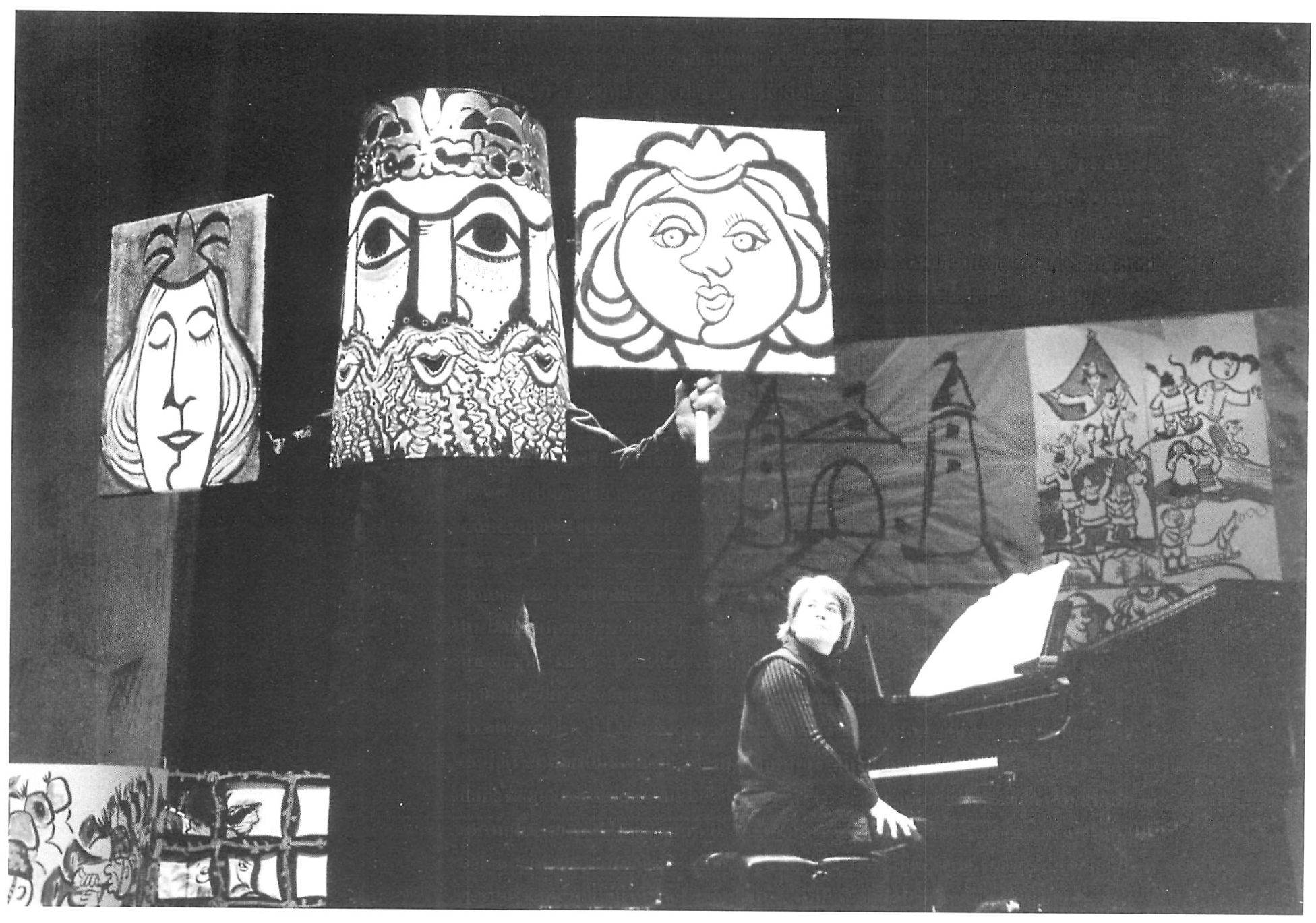

L'histoire du petit tailleur, musique de Tibor Harsanyi, texte des frères Grimm adapté par Louis-Dominique Lavigne, Smce Jeunesse, 1997 . Photo : Roy Hubler. 